\title{
La metodología Lean Startup: desarrollo y aplicación para el emprendimiento
}

\author{
Francisco Javier Llamas Fernández \\ European School of Leadership \\ franciscojllamas@hotmail.com \\ Juan Carlos Fernández Rodríguez ${ }^{2}$ \\ Universidad Antonio de Nebrija \\ jfernanr@nebrija.es
}

DOI: https://doi.org/10.21158/01208160.n84.2018.1918

Fecha de recepción: 12 de diciembre de 2017

Fecha de aprobación: 07 de marzo de 2018

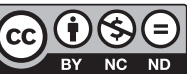

Cómo citar este artículo / To reference this article / Comment citer cet article / Para citar este artigo:

Llamas Fernández, F. J. y Fernández Rodríguez, J. C. (2018). La metodología Lean Startup: desarrollo y aplicación para el emprendimiento. Revista EAN, 84, (pp 79-95). DOI: https://doi.org/10.21158/01208160.n84.2018.1918

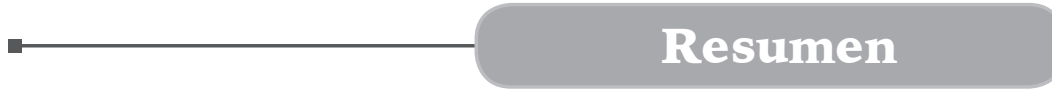

El presente artículo tiene como objetivo realizar una exposición sobre la aplicación de la metodología Lean Startup y revisar los distintos puntos y acciones que comprende. Lean Startup es una metodología con gran auge en el medio emprendedor que posibilita implementar negocios evitando desperdiciar tiempo, recursos y esfuerzos inútiles. Propone como paso previo a la creación de una empresa, lanzar una Startup, un experimento, que permita al emprendedor buscar un modelo de negocio rentable antes de crear una empresa. En la contabilidad de la metodología Lean Startup el principal beneficio a conseguir es el aprendizaje validado. Un aprendizaje empírico que parte de unas hipótesis que se validan a través de un producto con las características mínimas para facilitar al emprendedor los datos necesarios que permitan descubrir el modelo de negocio viable y dar el salto a la empresa, minimizando el miedo al fracaso que impide a muchos emprendedores convertir sus ideas en negocios.

\section{Palabras clave}

Emprendimiento, Startup, modelo de negocio, Lean Startup, metodologías ágiles, Producto Mínimo Viable (PMV), pivotar, iterar.

\footnotetext{
${ }^{1}$ Doctorando en Ciencias Sociales, Universidad Pontificia de Salamanca; Magister en Marketing Digital y Publicidad, Universidad Nebrija. Fundador y director académico, European School of Leadership (EUschool)

2 Doctor en Psicología por la Universidad Complutense de Madrid. En la actualidad es director del grado de psicología de la Universidad Antonio de Nebrija. Ha sido profesor en varias universidades españolas y profesor invitado en varias universidades latinoamericanas. ORCID: https://orcid.org/0000-0003- 3312-861X
} 


\title{
Lean Startup methodology: development and application to develop entrepreneruship
}

\begin{abstract}
This study aims at explaining the application of Lean Startup methodology and reviewing different points of views and actions that it involves. This is a methodology arising in entrepreneurial environments, facilitating the implementation of business and avoiding wasting time, resources and efforts. It proposes to launch a Startup as a former step to take in company creation. In fact, this is an experiment which allows the entrepreneur to find a profitable business model before starting a business. In the accountancy of the Lean Startup methodology, its main benefit is to reach validated learning. This empirical learning process originates from some validated hypothesis through a product with minimal features to provide the entrepreneur with necessary data which facilitates the discovery of a profitable business model and the start of the company, reducing the fear to failure that stops the process in which entrepreneurs turn their ideas into real business.
\end{abstract}

Key words: Entrepreneurship, Startup, business model, Lean Startup, fast methodologies, profitable mínimum product, pivoting, iterating.

\section{Méthodologie de Lean Startup: développement et application pour l'entrepreneuriat}

Résumé. Cet article présente la mise en place de la méthodologie Lean Startup et en étudie les différents principes et fonctionnements. La Lean Startup est une méthodologie de plus en plus utilisée dans le monde entrepreneurial car elle permet de gagner un temps précieux lors de l'implémentation d'une affaire et d'économiser aussi bien les ressources que les efforts inutiles. L'objectif de la Lean Startup est de procéder, avant la création formelle de l'entreprise, au lancement d'une Startup, d'une expérimentation, devant permettre aux entrepreneurs à la recherche d'un modèle d'affaires rentable de tester leur modèle avant de créer leur entreprise. Le principal avantage de la méthodologie Lean Startup est qu'elle permet un apprentissage validé de l'étape pré-entrepreneurial. Un apprentissage empirique partant d'hypothèses validées par un produit aux caractéristiques minimales offre à l'entrepreneur les éléments nécessaires pour découvrir un modèle d'affaire viable et franchir le pas de la création d'entreprise en réduisant la peur de l'échec qui empêche parfois les entrepreneurs de concrétiser leur modèle d'affaire.

Mots clefs: Entrepreneuriat, Startup, modèle d'affaire, Lean Startup, Viabilité Minimum du Produit (VMP), pivot, répétition.

\section{Metodologia Lean Startup: desenvolvimento e aplicativo para o empreendimento}

Resumo. O presente artigo tem como objetivo realizar uma exposição sobre o aplicativo da metodologia Lean Startup e revisar os diferentes pontos e ações que compreende. Lean Startup é uma metodologia com grande auge no médio empreendedor e que possibilita implementar negócios evitando desperdiçar tempo, recursos e esforços inúteis. Propõe como passo prévio à criação de uma empresa, lançar uma Startup, um experimento, que permita ao empreendedor procurar um modelo de negócio rentável dantes de criar uma empresa. Na contabilidade da metodologia Lean Startup o principal benefício a conseguir é a aprendizagem validada. Uma aprendizagem empírica que parte de umas hipóteses que se validam através de um produto com as características mínimas para facilitar ao empreendedor os dados necessários que permitam descobrir o modelo de negócio viável e dar o salto à empresa, minimizando o medo ao fracasso que impede a muitos empreendedores transformar suas ideias em negócios

Palavras-chave: Empreendimento, startup, modelo de negócio, Lean startup, metodologias ágeis, Produto Mínimo Viável $(P M V)$, girar, iterar. 


\section{Introducción}

$E^{1}$ emprendimiento siempre ha estado relacionado con exponerse a correr un gran riesgo. El riesgo puede, además, derivar en un fracaso, y esta situación siempre ha estado demonizada, no sin falta de razón, por las consecuencias que suele generar para el emprendedor; y es que la cruda realidad evidencia que el $75 \%$ de las Startups fracasan (Xavier, 2012).

Ries (2012), considerado el padre de la metodología Lean Startup, expone en El método Lean Startup cómo crear empresas de éxito utilizando la innovación continua. Afirma que el éxito de las startups no radica, como piensa mucha gente, en estar en el lugar correcto y en el momento adecuado, sino que el éxito de una startup se puede diseñar siguiendo un proceso correcto y esto significa que se puede aprender y enseñar.

La base del método Lean Startup radica en crear el producto que el cliente necesita y por el que está dispuesto a pagar, usando la cantidad mínima de recursos. El problema de muchos de los emprendedores que han fracasado es que crean un plan de negocio, consiguen financiación, desarrollan el producto y solo después de crearlo y lanzarlo la empresa obtiene retroalimentación de los clientes. Es el momento en el que muchos emprendedores aprenden que los clientes no necesitaban la mayoría de las características del producto o servicio.

Hace apenas unos meses, septiembre de 2017, se pudo conocer a través de periódicos de todo el mundo el fracaso de la Startup Juicero (Levin, 2017). Al fundador, Doug Evans, se le ocurrió desarrollar una idea con un modelo de negocio parecido al de las cápsulas de café de Nespresso. En su caso, se trataba de vender zumos naturales, en vez de café. Estos zumos naturales son empaquetados en bolsas para los cuales se creó un exprimidor que sacara el zumo fresco de la bolsa (Figura 1).

Figura 1. Exprimidor Juicero.

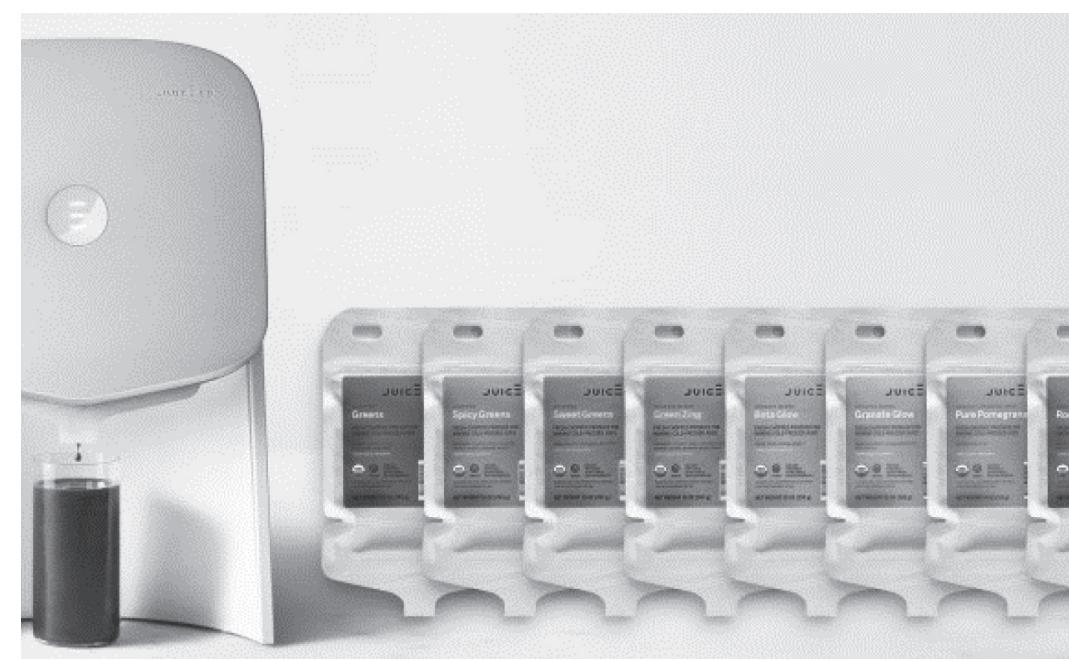

Fuente. Rodríguez (2017). 
Se lo contó a los inversores de Silicon Valley y entusiasmados con la idea le financiaron nada más y nada menos que USD 120 millones, con los cuales, Juicero, creó el exprimidor perfecto, prodigio de la ingeniería, con piezas hechas a la medida, lector de códigos QR para identificar la bolsa de zumo, un chip wifi para conectar el aparato a internet de las cosas y un procesador capaz de generar una potencia increíble para sacar hasta la última gota de la bolsa de zumo; en conclusión, un aparato que costaba USD 700. Hasta aquí todo perfecto, si no fuera porque este aparato parece no haber interesado a los potenciales clientes. Otro de los periódicos, titulaba la noticia: "Cierra Juicero, el inútil exprimidor de 700 dólares» (Jiménez, 2017).
Eric Ries, como muchos de los emprendedores, sufrió la experiencia de trabajar muy duro en la creación de productos que luego fracasaban en el mercado. Estaba cansado de perder tiempo e inversión, hasta que decidió experimentar con un nuevo enfoque que posteriormente denominaría Lean Startup (Ries, 2012).

La metodología Lean Startup se centra en un circuito de tres pasos que se deben recorrer en el menor tiempo posible y con la mínima inversión. Se comienza por crear un producto, se miden los resultados, y se aprende (Figura 2).

Figura 2. Circuito Lean Startup

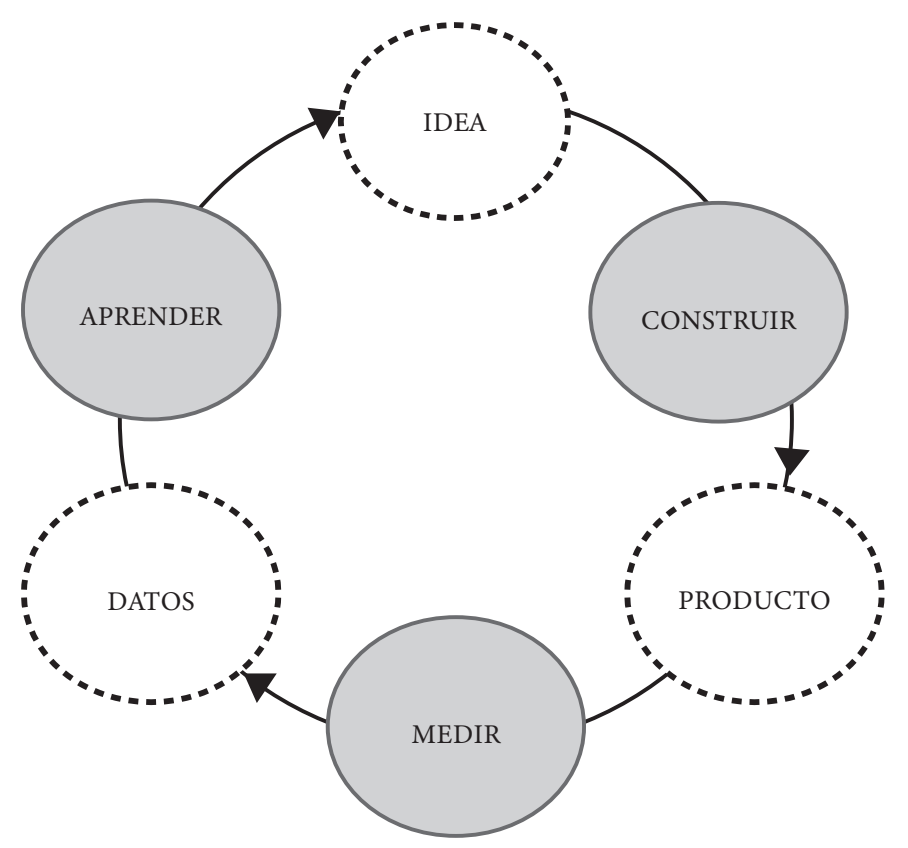

Fuente. Ries (2012). 
- Construir: cuando se lanza la Startup, no se cuenta con los suficientes datos como para crear un producto ajustado a las necesidades del cliente, por eso, lo ideal es crear un producto viable mínimo (PMV). Este producto debe ser una versión con las funcionalidades mínimas que permitan recoger la máxima cantidad de aprendizaje validado acerca de los clientes.

- Medir: el mayor reto en esta fase es medir cómo responden los consumidores y a partir de esos datos, tomar las decisiones apropiadas.

- Aprender: el circuito del proceso Lean Startup permite, sobre todo, aprender si es viable el negocio, seguir perseverando o de lo contrario, pivotar, es decir, reajustar sustancialmente las ideas que no están funcionando.

En síntesis, lo que propone la metodología Lean Startup es lanzar los negocios a través de este circuito ágil donde el emprendedor, una vez establecidas sus hipótesis y suposiciones, las va validando con un experimento, el PMV. El PMV permite saber con muy poca inversión si la idea que se está desarrollando tiene aceptación en el mercado; si se demuestra que responde al deseo del cliente, se irán incrementando sus funcionalidades $y$, por el contrario, si el PMV no encaja en el mercado, se deberá darle un nuevo enfoque al negocio -lo que se denomina pivotar-.

La razón de esta metodología es aprender en poco tiempo, invirtiendo los mínimos recursos. Lean Startup es una metodología dirigido a la puesta en marcha de ideas innovadoras, donde no se comienza creando una empresa, sino una Startup, entendida no como una empresa en pequeño, sino como «una institución humana diseñada para crear un nuevo producto o servicio bajo condiciones de incertidumbre extrema" (Ries, 2012, p. 39). El objetivo de las Startup, señala Ries «es averiguar qué debe producirse, aquello que los consumidores quieren y por lo que pagarán, tan rápidamente como sea posible» (p. 32). La Startup es un experimento en el que la pregunta no es ¿puede crearse este producto? sino ¿debería crearse este producto?

Otra de las autoridades en la metodología Lean Startup, Steve Blank, mentor de Eric Ries, define una Startup como «una organización temporal en busca de un modelo de negocio rentable, escalable y que puede repetirse. Al principio, el modelo, de una Startup, es un lienzo con ideas y suposiciones y con muy poco conocimiento sobre esos clientes» (Blank y Dorf, 2012, p. 29). En la práctica, comenta el autor, las Startups comienzan con un conjunto de hipótesis, supuestos, que en su mayoría serán erróneas. Por eso, el gran peligro de muchos emprendedores es que centran en la ejecución del producto sin haber validado las hipótesis, viéndose abocados al fracaso.

Ash Maurya, reconocido asesor y mentor de Startups en su famoso libro Running Lean: cómo iterar de un plan $A$ a un plan que funcione, muestra las tres fases que atraviesa la Startup (Maurya, Ries y Marqués, 2014, p. 34) (Figura 3).

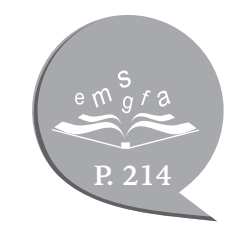


Figura 3. Fases por las que atraviesa una Startup

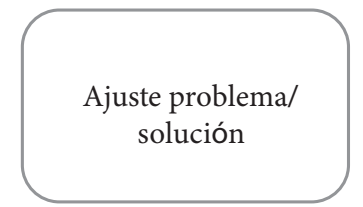

Fase 1

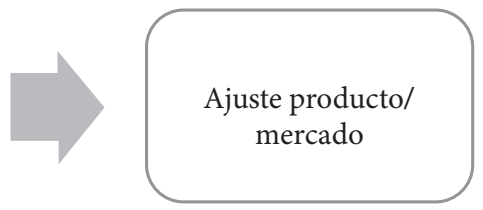

Fase 2

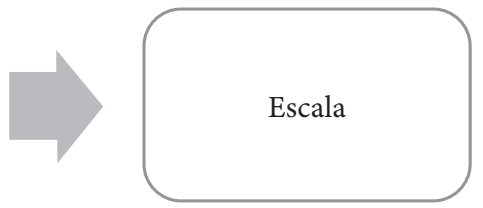

Fase 3

Fuente. Adaptado de Maurya (2014).

- Fase 1: Ajuste problema/solución: consiste en determinar si realmente se tiene un problema que merezca la pena solucionar antes de invertir tiempo y esfuerzo para desarrollar una solución. Se puede resumir esta fase en dos preguntas: ¿está interesado el cliente realmente en el producto? y ¿pagaría por ello?

- Fase 2: Ajuste producto/mercado: se debe comprobar que se consigue tracción, es decir, que la gente demanda ese producto.
- Fase 3: escala: es necesario centrarse en la aceleración del crecimiento.

Las empresas establecidas ejecutan modelos de negocio donde se conoce al cliente, sus problemas y las características de los productos que demandan, mientras que las Startups necesitan operar en forma de búsqueda, validando sus hipótesis y aprendiendo de los resultados para buscar el modelo de negocio escalable y rentable.

\section{Antecedentes que fundamentan la metodología Lean Startup}

$\mathbf{R}_{\text {ies (2012), quien tiene registrada la }}$ que muchos de los productos que se crean, después de un gran esfuerzo, fracasan por no tener acogida en el mercado. En su experiencia como emprendedor, descubre que existen varias metodologías en diversos sectores que, aplicándolas al emprendimiento, de una forma innovadora, pueden ayudar a generar éxito. De dichas metodologías, caben destacar: Lean Manufacturing, desarrollo ágil y desarrollo de clientes (Ries, 2009).

\subsection{Lean manufacturing}

En el sistema de producción de Toyota, denominado Lean Manufacturing, Ries encontró una de las bases fundamentales que, aplicadas a la innovación, derivaría en la metodología Lean Startup. Lean Manufacturing o producción ajustada, como se denominó en un principio, es un modelo que trata de entregar el máximo valor a los clientes, utilizando el mínimo de los recursos necesarios (Womack, Jones y Roos, 1993). 


\subsection{Desarrollo ágil}

Ries, por su formación y desempeño como ingeniero de desarrollo de software, incorpora las metodologías de desarrollo ágil en su metodología Lean Startup. Los métodos ágiles -adaptativos, en contraposición con los métodos clásicos denominados en cascada, predictivos-, tienen la visión general del producto o servicio, pero sin especificar el resultado final, ya que se generarán unas hipótesis, que darán lugar a un prototipo del producto que se usará para explorar el mercado y validar dichas hipótesis o modificarlas, en un continuo ensayo de prueba y error que va adaptando el producto.

Con los métodos ágiles, se desarrollan proyectos en unidades de tiempo llamadas iteraciones. Cada iteración incluye un ciclo de desarrollo del producto que se entrega en un periodo de tiempo y que va añadiendo funcionalidades, de tal forma que las entregas se van convirtiendo en demos que permiten evaluar la funcionalidad del producto en colaboración con el cliente e incorporar cambios continuamente sin esperar la entrega final de dicho producto (Álvarez, De las Heras y Lasa, 2012). Este modelo busca la agilidad a través de valorar la interacción entre las personas, la colaboración y la flexibilidad al cambio (Fowler, 2005).

\subsection{Desarrollo de clientes}

Blank critica la forma tradicional de crear empresas centradas en el desarrollo del producto, donde se conceptualiza la idea del producto o servicio: se desarrolla, se prueba y se lanza al mercado (Blank y Dorf, 2013). El problema es que la mayoría de las Startups fracasan porque no tienen clientes. Frente a este enfoque, Blank (2013) propone centrar el foco en el cliente. El proceso de desarrollo de clientes comienza con el establecimiento de unas hipótesis, unas suposiciones, que deben validarse desde el contacto directo con estos. Lo primero que se debe hacer es conocer si el cliente realmente tiene una necesidad y está dispuesto a pagar por ella; y lo segundo, a través de un mínimo producto viable, ver si realmente demandaría este producto. Posteriormente, se procede a hacer la transición de lo que ha sido una Startup -enfocada en el aprendizaje hacia la creación de una compañía enfocada en la ejecución del negocio- que se ha encontrado viable (Figura 4).

Figura 4. Fase de desarrollo de clientes

Búsqueda

Ejecución

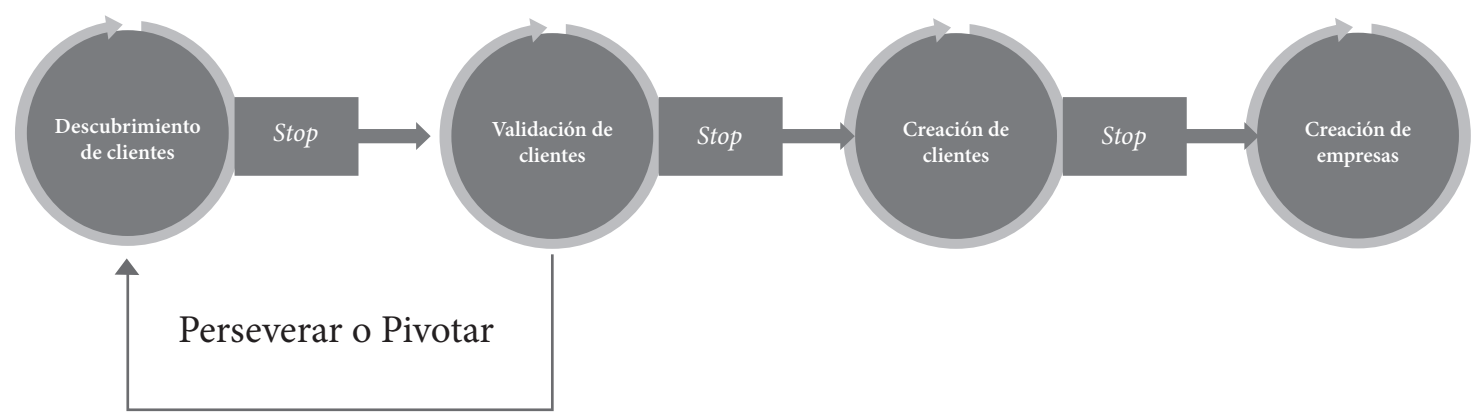

Fuente. Blank y Dorf (2013). 
En síntesis, se podría decir que los tres pilares esenciales en los que se fundamenta la metodología Lean Startup son: i) crear valor para el cliente, entendido como la eliminación de desperdicio, ofreciéndole lo que realmente demanda, con la calidad correspondiente; ii) desarrollar el producto desde la óptica del cliente, aprendiendo y validándolo desde el continuo contacto con él; y iii) desarrollar ese producto de una forma ágil, flexible, iterativa, incorporando nuevas funcionalidades a medida que se desarrolla.

\section{La metodología Lean Startup}

$E_{\text {central de la metodología Lean Startup. }}^{\text {lcircuito crear-medir-aprender es el núcleo }}$ (Ries, 2013). Una Startup crea productos, mide resultados y aprende de ellos. En otras palabras, es un proceso iterativo de transformación de ideas en productos, medición de la reacción y comportamiento de los clientes frente a los productos y el aprendizaje o bien porque se persevere o bien porque se pivote. Este proceso se repite de forma continuada.

La secuencia se inicia con el primer elemento indispensable en el planteamiento del negocio: las hipótesis, que no son sino las suposiciones sobre las que el emprendedor fundamenta la viabilidad de su idea. Para comprobar dichas hipótesis, el emprendedor se ayudará de un PMV con el que experimentará, y sobre todo, aprenderá de su público objetivo qué aceptación tiene su oferta. Pero para que este aprendizaje sea riguroso y fundamentado, es indispensable medirlo y como consecuencia, tomar las decisiones oportunas que implicarán correcciones sobre las hipótesis iniciales, reestructurando el modelo de negocio, lo cual se denominará pivotar. Si por el contrario los datos aconsejan persistir, la iteración continuará con la creación de versiones incrementales del producto que se puedan ir validando desde la experiencia del cliente.

El objetivo principal de aplicar este metodología es obtener un aprendizaje validado, saber qué elementos de la estrategia funcionan y conocer qué es lo que quiere el consumidor. Este aprendizaje permitirá medir el progreso de la Startup y descubrir si el modelo de negocio que se busca es viable, rentable y escalable; características fundamentales que debe tener una Startup antes de convertirse en una empresa (Figura 5).

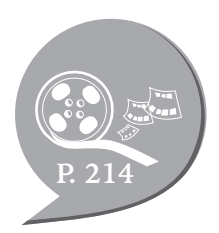


Figura 5. Secuencia en el planteamiento de negocio

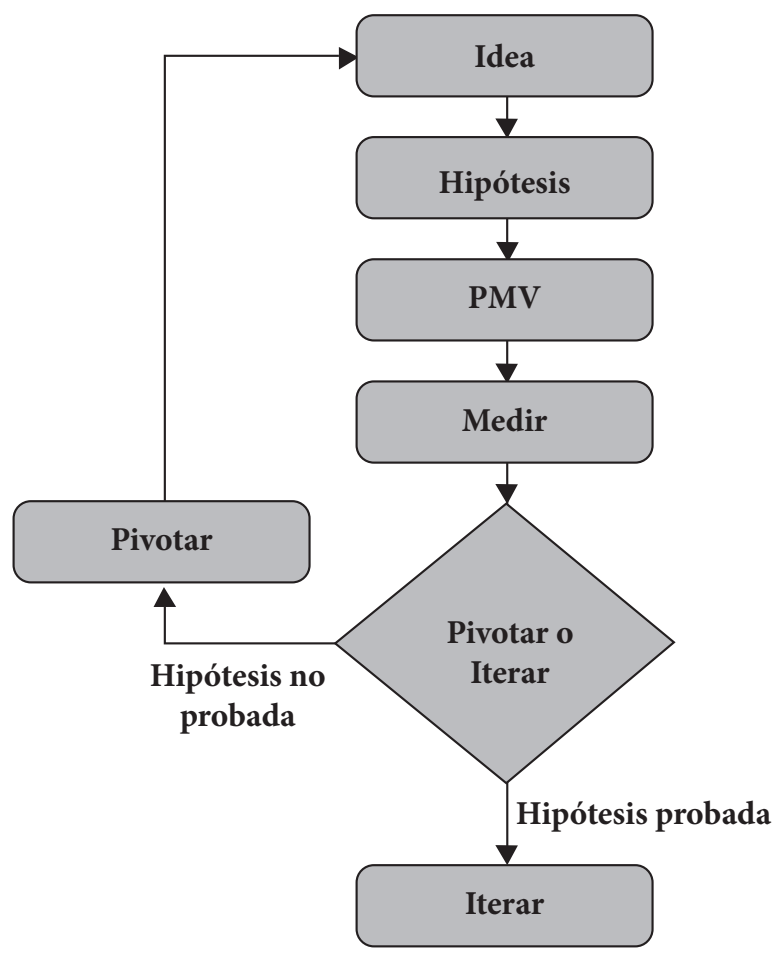

Fuente. Elaboración propia.

\subsection{Validación de hipótesis}

Antes de llevar a cabo una idea, siempre se tiene una intuición, un presentimiento de que va a ser exitosa, de lo contrario, no valdría la pena embarcarse en esa aventura. Pero no todas las ideas son exitosas, al contrario, son pocas las ideas que triunfan. Lo duro de emprender en cualquier proyecto, sobre todo cuando es algo innovador, es la incertidumbre que conlleva. Los proyectos se plantean sobre la base de una serie de suposiciones, asunciones, hechos no probados, que en la metodología Lean Startup se denominan hipótesis y que es muy importante que se comprueben antes de lanzar el negocio y desperdiciar tiempo y recursos (Ries, 2013).
Javier Megias (2013), reconocido emprendedor, tiene en su blog una definición de hipótesis, aplicada a los negocios, que es muy ilustrativa: «Son aquellos hechos que consideramos como ciertos en nuestro modelo de negocio pero que realmente no estamos seguros al $100 \%$ \%.

Y Blank y Dorf señalan que «en este ámbito, hipótesis es solo una palabra elegante para referirse a las suposiciones" (2013, p. 88). Los autores proponen que, a la hora de emprender, se parta de un modelo de negocio que ayude a establecer las hipótesis y probar los supuestos de los que parte el emprendedor y para ello recomiendan el lienzo o Canvas de Osterwalder, como punto de partida (Figura 6). 
Figura 6. Modelo de lienzo de Osterwalder

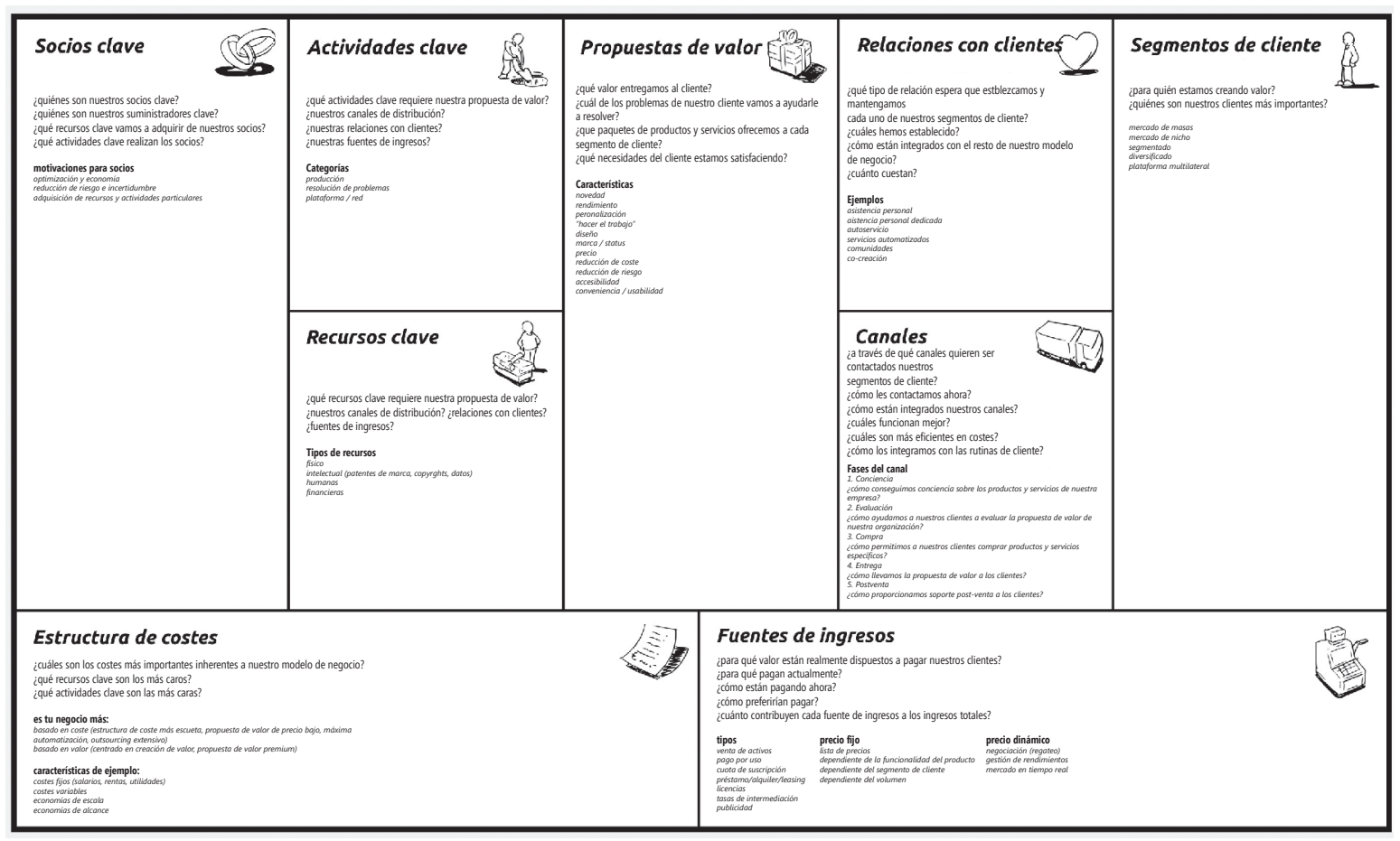

Fuente. Osterwalder y Pigneur (2011).

Como se pudo ver, Osterwalder y Pigneur en Generación de modelos de negocio (2011), facilitan una herramienta muy interesante para testar las hipótesis más arriesgadas que ponen en juego la viabilidad del negocio. Se siga esta herramienta o cualquier otra, los creadores del negocio necesitan transformar las hipótesis o los supuestos en hechos tan pronto como sea posible, saliendo a la calle, preguntando a los clientes si las hipótesis son correctas y rápidamente cambiar aquellas que estuvieran equivocadas (Blank y Dorf, 2013).

\subsection{Producto viable mínimo}

Una de las mejores formas de validar las hipótesis es a través de la construcción de un $\mathrm{PMV}$, que viene a ser la versión del producto que permite dar la vuelta entera al circuito de crear-medir-aprender, con un mínimo esfuerzo y en un mínimo tiempo. El PMV es una de las técnicas más importantes del Lean Startup, que Ries define como «la versión de un nuevo producto que permite a un equipo recoger la máxima cantidad de aprendizaje validado, acerca de sus clientes, con el mínimo esfuerzo» (2009, p. 91).

Para Blank y Dorf (2013), el PMV consiste en «una síntesis concisa del conjunto más pequeño posible de características que podrían funcionar como producto independiente resolviendo al menos el problema principal y demostrando el valor del producto» (p.136).

El PMV ayuda a los emprendedores a empezar con el proceso de aprendizaje lo más rápidamente posible. Su objetivo es probar las hipótesis fundamentales del negocio.

El primer producto de una Startup no está dirigido a satisfacer al público en general. Las Startups no pueden permitirse crear un producto que contenga todas las 
características desde el inicio y, por eso, los primeros esfuerzos se centran en un grupo muy pequeño que algunos llaman evangelistas, early adopters o pioneros. Son aquellos clientes que quieren ser los primeros y están dispuestos a adoptar el producto o servicio en sus fases iniciales. Clientes visionarios, con interés por el producto (Rogers, 2003).

Para construir un producto viable mínimo, no hay una fórmula exacta. Lo importante es que ese producto mínimo haga posible al emprendedor conocer su viabilidad; dicho de forma muy sencilla, permitir saber si el producto tiene futuro o no. Por eso, es muy interesante el apartado de experimentos de viabilidad de Cooper y Vlaskovits en El emprendedor Lean: cómo los visionarios crean nuevos productos, desarrollan proyectos innovadores y transforman los mercados (2014, p. 183), en el que plantean que en el diseño de un PMV es fundamental conocer la viabilidad antes de construir el producto. Lo ilustran con un sencillo ejemplo:

Si quieres abrir un restaurante, ¿no deberías comprobar si a la gente le gusta este tipo de comida? Antes de lanzar un primer intento de producto al mercado, que crea valor a un segmento concreto de clientes, es importante realizar experimentos que validen los aspectos del modelo de negocio.

De estas pruebas algunas son populares: landing page, la prueba del conserje, la creación de prototipos, la prueba del crowdfunding o la prueba del Mago de Oz (Macías, 2017). Todas ellas son interesantes, dependiendo lo que se quiera probar, por ejemplo, en la prueba del Mago de $\mathrm{Oz}$ se trata de proporcionar un lado visible del producto al cliente para que pueda comprarlo, pero se satisface el propósito del producto de forma manual, como prueba, antes de lanzarlo online. Es famoso el ejemplo de Zappos, que se originó utilizando esta técnica. En Delivering happiness: A path to profits, passion, and purpose, Hsieh (2011) cuenta cómo un día Nick Swinmurn estando en un centro comercial no podía encontrar un par de botas Airwalk Desert y pensó: «¿Por qué no montar una tienda online de calzado?». Tras lo cual se acercó a algunas tiendas locales de calzado y les propuso sacar fotos y ponerlas en una sencilla web y, si la gente lo solicitaba, se los compraría al mismo precio a la tienda de calzado. Nick corría a la zapatería local y compraba el artículo y se lo enviaba al cliente. En 1999, estaba utilizando esta prueba. Posteriormente, con financiación, y no exento de dificultades, desarrolla un portal de comercio electrónico, Zappos, que diez años después sería comprado por Amazon por la cantidad de USD 1200 millones.

Exovite es un ejemplo muy ilustrativo de Startup que, aprovechando la impresión 3D, diseñó, como producto mínimo viable, una férula de plástico para sustituir la escayola o yeso que tradicionalmente deben llevar los pacientes cuando sufren un traumatismo. Después de probarlo en numerosos pacientes, con la asesoría y acompañamiento de médicos expertos, Exovite ha dejado de ser una Startup para convertirse en una empresa de biotecnología especializada en sistemas de rehabilitación (Figura 7). 
Figura 7. Férula, producto viable mínimo de Exovite

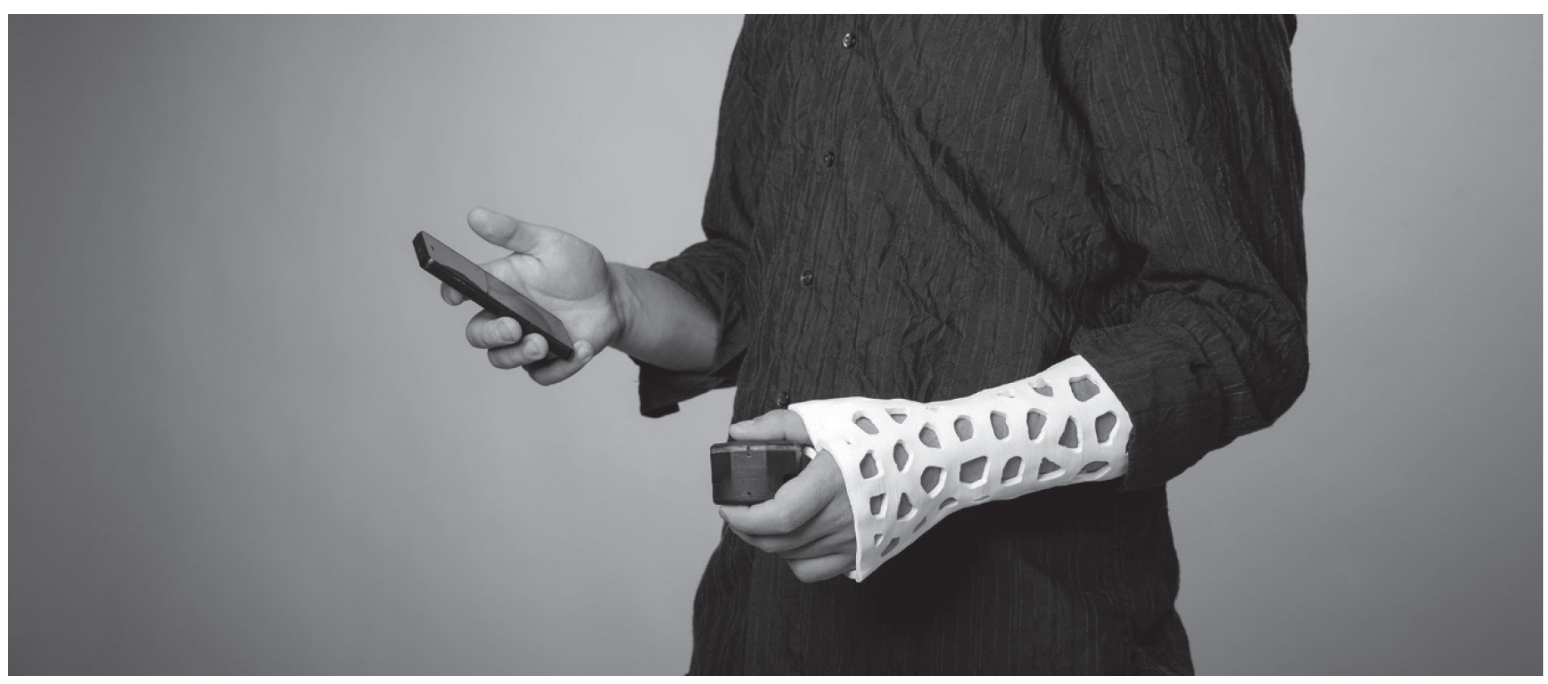

Fuente. Exovite (s. f.).

\subsection{Medir: Lean Analytics}

En general, las empresas usan la contabilidad para medir su objetivo principal: el crecimiento. Este depende básicamente de la rentabilidad que se obtiene del cliente, los costes que supone obtener a dicho cliente y la tasa de repetición en la compra.

La contabilidad de una Startup es muy distinta, pues el objetivo es demostrar que se está aprendiendo a construir un negocio sostenible. Es lo que llama Ries la contabilidad de la innovación (2012, p. 135) y que explica en tres etapas:

- Usar un producto mínimo viable para recopilar los datos reales, sobre en qué punto se encuentra la empresa en el momento actual.

- La Startup hace todos los cambios y las optimizaciones del producto.

- La Startup toma la decisión de perseverar porque está aprendiendo apropiadamente y por tanto haciendo un buen progreso o si el equipo concluye que necesitan cambios, deben pivotar comenzando el proceso de nuevo.

Para poder llevar a cabo este proceso de la contabilidad de la innovación, del aprendizaje y tomar decisiones rigurosas, es necesario ser capaces de medirlo.

Cada modelo de negocio y cada tipo de producto requiere un servicio de métricas diferente. El objetivo de los negocios es que los clientes compren un producto o un servicio y esto implica seguir la ruta de los pasos que sigue el cliente hasta que genera ingresos a la Startup.

La forma más clara de seguir esta ruta es el embudo de conversión definido por las métricas piratas. Estas fueron acuñadas por Dave McClure (2010), miembro integrante de los fundadores de 500 Startups, una de las principales aceleradoras de Startups de los Estados Unidos. Se definen así porque las iniciales de los cinco bloques del embudo en inglés son AARRR, como el supuesto berrido de un pirata -acquisition, activation, retention, referral, revenue- (Figura 8). 
Figura 8. Métricas piratas, embudo de conversión

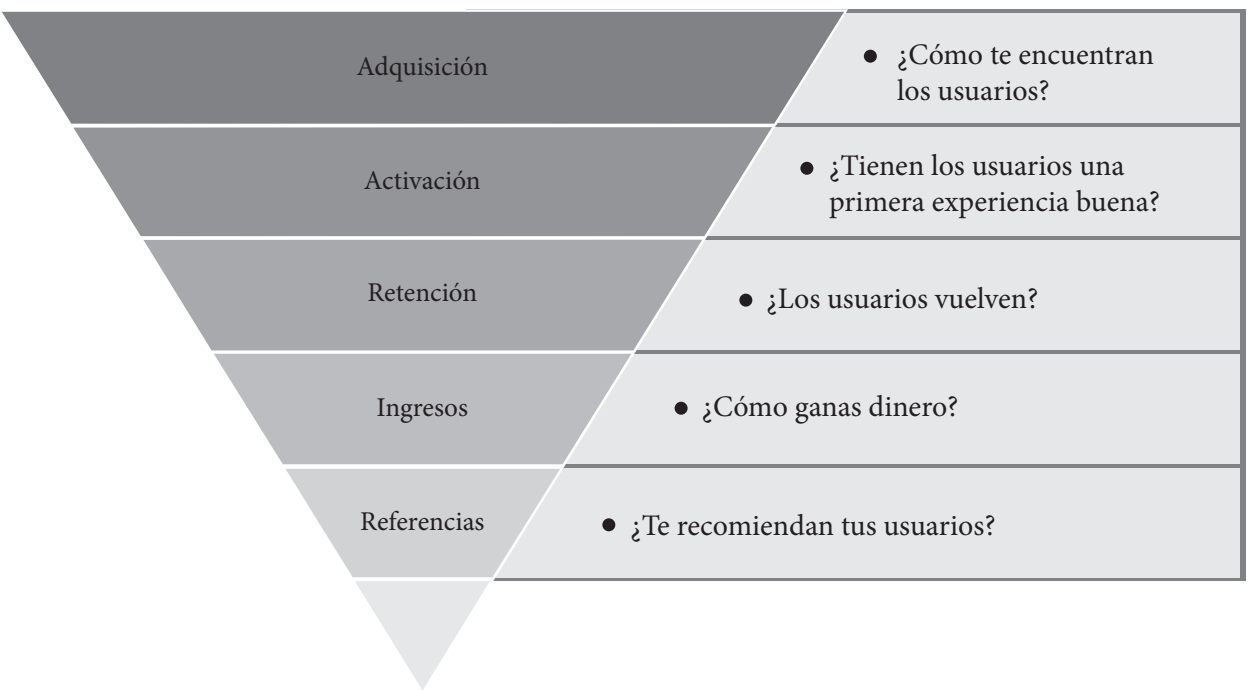

Fuente. Adaptado de McClure (2010).

¿Por qué el empleo de métricas piratas? Vicent Esteve (2015), asesor de Startups, apunta un doble motivo: por un lado, las métricas piratas son un sistema de análisis cuantitativo de un modelo de negocio comúnmente aceptado por la mayor parte de Startups; y por otro, los costes de ir llevando al cliente potencial a cerrar una venta vinculan el modelo de generación de ingresos con una de las partes más importantes de la estructura de costes de Startups, que es la inversión en captación de clientes.

De forma práctica, el embudo de las métricas piratas tomado de McClure (2010) seguiría la siguiente secuencia:

- Adquisición: responde a la pregunta ¿cómo llega el cliente o usuario a conocer la oferta del negocio? Por tanto, se deben identificar los canales de adquisición y los costes asociados, en el caso de que se haya hecho una campaña SEM -search engine marketing 'marketing en buscadores'- o un mailing u otro tipo de actividades offline, como pudiera ser el caso de una feria.
- Activación: según Megias (2013), se trata de convertir a un potencial interesado en un cliente potencial. Mide el porcentaje de potenciales interesados que han hecho la acción necesaria para convertirse en cliente potencial. Por ejemplo, rellenar un formulario, darse de alta en una plataforma, registrarse en una app. Indica la capacidad de despertar interés en el interesado para que vuelva a contactarnos. Está muy relacionada con la experiencia que haya tenido. Se calcula dividiendo los usuarios que se consideran activados, los que descargaron la app, entre los usuarios adquiridos.

- Retención -engagement-: por decirlo de forma muy coloquial, se trata de lo enganchado que está el cliente. ¿Vuelve el usuario? ¿Recurre? Es importante entender el porqué de la fidelidad de cliente o su abandono.

- Referencia: esta métrica indica el número de clientes que vienen por recomendación o por viralidad. Usualmente, el cliente referido no tiene un coste y eso influye 
directamente en la disminución del coste de adquisición de clientes.

- Conversión: este indicador permite saber a qué porcentaje de clientes se ha conseguido vender del total del público al que se ha logrado interesar. Es una métrica muy importante, porque permite conocer al emprendedor si es capaz de monetizar, y por tanto, rentabilizar su modelo de negocio. Para calcular el porcentaje de conversión, se debe dividir el número de clientes convertidos, que han comprado, entre los clientes adquiridos.

A la hora de medir es fundamental saber qué métricas son las más importantes para el devenir de la empresa. Para Justo Hidalgo, fundador de 24 symbols, "son aquellas sin las que no podemos tomar decisiones en un negocio más allá de la intuición» (2013, p. 4). El número de usuarios, comenta, que visitan una web puede ser una métrica muy importante al principio, pero seguro que en un determinado momento es mucho más importante medir y actuar sobre el porcentaje de conversión.

No hay una métrica concreta que se deba seguir desde la concepción de la idea del negocio, sino que en cada momento se pondrá el foco en una métrica a la que se le concederá más importancia. En el mundo de los datos, aconsejan Croll y Yoskovitz (2014, p. 82), es importante elegir una métrica clave para el momento en el que se encuentre la Startup, y a esa métrica la bautiza como «UMVI, la única métrica que verdaderamente importa». Será la métrica que ayude a encontrar el producto correcto y el hueco de mercado antes de que se acabe el dinero.

\subsection{Pivotar o perseverar}

Todos los elementos que se han desarrollado hasta el momento como aspectos importantes dentro de la metodología Lean Startup: establecer hipótesis, crear un producto mínimo viable, medirloy aprender deello, tienen sentido en cuanto responden a la siguiente pregunta: ¿se están haciendo progresos suficientes como para creer que la hipótesis es correcta o se debe hacer algún cambio importante? Este cambio, según Rice, se llama pivote: una corrección estructurada diseñada para probar una nueva hipótesis básica sobre el producto, la estrategia y el motor de crecimiento (2012, p. 167). Y prosigue, un pivote requiere que se mantenga un pie anclado en lo que se ha aprendido hasta el momento, mientras se hace un cambio fundamental en la estrategia para buscar un mayor aprendizaje validado.

Blank, por su parte, para ilustrar lo que es un pivote, recurre al lienzo del modelo de negocio de Osterwalder y Pigneur, ya aludido, y explica en su blog el significado de pivote como una modificación significativa de uno o varios componentes de aquel. Un pivote, comenta el experto, no es solo cambiar un producto, un pivote significa una modificación de cualquiera de los factores del modelo de negocio: la propuesta de valor, la generación de ingresos, el canal, la segmentación o cualquiera de los componentes del lienzo (Blank, 2014).

Por tanto, se pivota como consecuencia de un aprendizaje en el que el emprendedor se da cuenta de que tiene que darle un nuevo giro a su negocio si no quiere quedarse estancando, consumiendo los recursos, mientras el negocio corre el peligro de no crecer o incluso morir. 
La pregunta obligada es ¿cuándo se debe pivotar? Rosa Alnasser (2015), quien dirige Lean Monitor, una herramienta de simulación para entrenar a emprendedores en el diseño de su Startup, comenta que se debe hacer en el mismo momento en el que el emprendedor se da cuenta de que su planteamiento no es válido. La clave, argumenta, radica en adquirir las evidencias empíricas necesarias para poderlo hacer cuanto antes y evitar la pérdida de tiempo y dinero, por eso, es tan importante identificar las hipótesis y hacer experimentos con ellas con el objetivo de validarlas o invalidarlas.
Como conclusión, se podría afirmar que el pivote responde a la necesidad de ser productivo e implica replantearse lo que se ha venido haciendo en el negocio para encontrar una dirección más positiva. Lo importante es haber aprendido a tiempo y sin abandonar el negocio, con un pie anclado, saber darle otra orientación. Cuando los datos que se van obteniendo aconsejan persistir, se continúa con la creación de versiones incrementales del producto o servicio que la experiencia del cliente irá validando.

\section{Conclusiones}

T a metodología Lean Startup propone el Llanzamiento de los negocios desde un aprendizaje que se va validando a través de un circuito. Se inicia con la concepción de un producto o servicio, de forma experimental, en el que se mide su encaje en el mercado y la aceptación por parte de los clientes más interesados; de esa realimentación, se obtiene un aprendizaje que permite seguir desarrollando dicho producto o servicio de una forma iterativa, ya sea persistiendo en el incremento de las funcionalidades del servicio o producto, ya sea estableciendo una serie de cambios o pivotes que permitan su viabilidad, tras lo cual se logra un modelo de negocio que funcione, sin desperdiciar recursos.

La metodología se ha desglosado siguiendo una secuencia recomendable a la hora de poner en marcha un negocio:

- Validación de hipótesis: validar las suposiciones de las que se parte, ya que no dejan de ser asunciones las cuales solo se deberían confirmar contrastadas con el mercado y el cliente. Para ello, se recomienda usar alguno de los lienzos de modelo de negocio que han desarrollado los expertos o crear un propio planteamiento de modelo de negocio.

- Creación de un producto mínimo viable: crear un producto con unas características mínimas que aporte un valor al cliente y que permita obtener la mayor información posible.

- Métricas: establecer elementos cuantificables que permitan medir el rendimiento esperado para poder tomar las decisiones oportunas.

- Pivotar o perseverar: el aprendizaje obtenido, a través de iteraciones, permitirá seguir incrementando lo que funciona, perseverar o implementar los cambios que se infieren de los datos obtenidos, pivotar.

Probablemente, Doug Evans, fundador de Juicero, y su equipo hicieron un plan de negocio $y$ se centraron en ejecutarlo, 
creando una empresa, pero el choque con la realidad dio al traste con una gran inversión. La aplicación de la metodología Lean Startup, en su caso, tal vez no hubiera supuesto el éxito del aparato exprimidor, pero sí hubiera evitado un gran desperdicio de tiempo, dinero y dedicación.

La metodología Lean Startup hubiese comenzado por validar las hipótesis; el problema es que esto no se hizo. No «salió de su oficina" para validar si los futuros clientes padecían realmente un problema que él estaba solucionando con su aparato; $y$ más aún si esos clientes estaban dispuestos a pagar por ello. En este ejercicio de validar las hipótesis, se hubiera dado cuenta de que muchos de los fanáticos de los zumos naturales prefieren hacerlos directamente en casa con sus propias licuadoras.

Además, creó directamente un producto final, cuando un producto mínimo viable le hubiera permitido conocer las necesidades del cliente y su disposición a adoptarlo. El PMV hubiera arrojado una serie de métricas, indispensables para tomar las decisiones oportunas: pivotar o persistir a través de versiones incrementales del producto.

Por tanto, si antes de crear una empresa y solicitar una gran financiación hubiese lanzado una Startup enfocada en el aprendizaje, hubiera podido validar el modelo de negocio, para posteriormente proceder a la creación de una compañía viable, rentable y escalable.

La metodología Lean Startup, sin duda, ofrece una gran oportunidad para abordar negocios innovadores y minimizar la incertidumbre, el desperdicio de recursos y el riesgo elevado que suele conllevar el emprendimiento.

\section{Referencias}

Álvarez, A., De las Heras, R. y Lasa, C. (2012). Métodos agiles y scrum. Madrid: Anaya.

Alnasser, R. (2015). ¿Qué significa pivotar un proyecto o Startup? Recuperado de http://blog.leanmonitor. com/es/que-significa-pivotar-un-proyecto-oStartup/

Blank, S. (2013). The four steps to the epiphany: Successful strategies for products that win. BookBaby.

Blank, S. (2014). Do pivots matter? Recuperado de https://steveblank.com/2014/01/14/whats-apivot/

Blank, S. y Dorf, B. (2013). El manual del emprendedor. Barcelona: Gestión 2000.

Cooper, B. y Vlaskovits, P. (2014). El emprendedor lean: cómo los visionarios crean nuevos productos, desarrollan proyectos innovadores $y$ transforman los mercados. España: Universidad Internacional de La Rioja.

Croll, A. y Yoskovitz, B. (2014). Lean analytics. España: Universidad Internacional de La Rioja.

Esteve, V. (2015). Métricas piratas y plan financiero en Startups. Recuperado de http://vicentesteve.com/ metricas-piratas-y-plan-financiero-en-Startups/

Exovite (s. f.). Innovación tecnológica en herramientas $y$ procedimientos médicos en Tele-RHB, E-Health $y$ M-Health. Recuperado de http://www.exovite.com/ es/exovite-es/

Fowler, M. (2005). Predictive versus adaptive. Recuperado de http://www.martinfowler.com/articles/ newMethodology.html\#PredictiveVersusAdaptive

Hidalgo, J. (2013). Métricas. En M. López de Ávila y J. A. de Miguel (eds.), El estado del arte del emprendimiento lean en España. España Lean Startup.

Hsieh, T. (2011). Delivering happiness: A path to profits, passion, and purpose. New York. Hachette Book Group.

Instituto Lean Management (s. f.). ¿Que es Lean? Recuperado de http://www.institutolean.org/es/ acerca-de/que-es-lean/70-definicion 
Jiménez Cano, R. (2017, septiembre 2). Cierra Juicero, el inútil exprimidor de 700 dólares: la Startup que defraudó con tecnología innecesaria despide a todos sus empleados. El País. Recuperado de https://elpais.com/tecnologia/2017/09/01/ actualidad/1504295056_379023.html

Levin, S. (2017). Squeezed out: Widely mocked Startup Juicero is shutting down. Recuperado de https:// www.theguardian.com/technology/2017/sep/01/ juicero-silicon-valley-shutting-down

Macías Rodríguez, M. (2017). El camino para innovar: cómo pasar de la idea al modelo de negocio creando valor para tus clientes. Barcelona: Deusto.

Maurya, A., Ries, E. y Marqués, M. (2014). Running Lean: cómo iterar de un plan $A$ a un plan que funcione. España: Universidad Internacional de La Rioja.

McClure. D. (2010). Startup metrics for pirates. Recuperado de https://es.slideshare.net/ dmc500hats/Startup-metrics-for-pirates-longversion

Megias, J. (2013, mayo 28). Validation Board, una herramienta para gestionar tus hipótesis [Entrada blog]. Recuperado de http://javiermegias.com/ blog/2013/05/validation-board-herramientavalidar-hipotesis-pivotar/
Osterwalder, A. y Pigneur, Y. (2011). Generación de modelos de negocio. Barcelona: Planeta.

Ries, E. (20093). Minimum Viable Product: A guide. Recuperado de http://www.Startuplessonslearned. com/2009/08/minimum-viable-product-guide. html

Ries, E. (2012). El método Lean Startup. Barcelona: Deusto.

Rodríguez, A. (2017). Juicero: de 100 a 0 en cuestión de segundos. Recuperado de https://hipertextual. com/2017/09/origen-caida-juicero

Rogers, E. (2003). Diffusion of innovations. New York: Free Press.

Womack, J. P., Jones, D. T. y Roos, D. (1993). La máquina que cambió el mundo. Madrid: McGraw-Hill.

Xavier, J. (2012, septiembre 21). 75 \% of Startups fail, but it's no biggie. Recuperado de https://www. bizjournals.com/sanjose/blog/2012/09/mostStartups-fail-says-harvard.html 